UDC $536.46+536.24$

\title{
COMBUSTION OF ALUMINUM POWDER-AIR SUSPENSION IN A SWIRL FLOW
}

\author{
Moiseeva K.M. ${ }^{*}$, Krainov A.Yu. ${ }^{1}$, Krainov D.A. ${ }^{2}$ \\ ${ }^{1}$ National Research Tomsk University, Tomsk, Russia, Moiseeva_KM@t-sk.ru \\ ${ }^{2}$ National Research Tomsk Polytechnic University, Tomsk, Russia
}

\begin{abstract}
The article is devoted to the numerical solution of the problem of the combustion of powder metal fuel in a combustion chamber with swirling flow. A physico-mathematical model of the flow of an air suspension of aluminum powder in a swirling flow in a cylindrical combustion chamber with a sudden expansion is presented. The physical and mathematical formulation of the problem is based on the approaches of the mechanics of two-phase reacting media. The solution was carried out using the arbitrary discontinuity decay method. The results of a numerical parametric study of the features of the combustion of an air suspension of aluminum powder depending on its composition, the axial flow rate of the mixture at the entrance to the combustion chamber, and the value of the swirl speed are shown.
\end{abstract}

Keywords: powder metal fuel, flow swirling, mechanics of two-phase reacting media, aluminum combustion.

\section{Introduction}

Aluminum is one of the most popular metals currently used in powdered metallic fuels. The studies [1 - 6] propose using aluminum powder as an independent energy carrier in ramjet rocket engines. The studies provide the results of the experimental and numerical investigation on the influence of the mixture parameters and characteristics of the combustion chamber on the combustion of the aluminum powder-air suspension (ALAS) in the airflow. The research [1] is devoted to the ignition, combustion and flame stabilization processes of the aluminum-air mixture in a high-speed airflow. The study summarizes the controlling methods of the highly dispersed aluminum combustion in the direct-flow combustion chamber. The paper [2] presents the physical-mathematical model and the results of the numerical modelling of the aerodynamics and combustion of the aluminum-air mixture in a swirling flow inside an axisymmetric channel with a sudden expansion. The probability of the reverse flow formation in the chamber is shown. The studies $[3,4]$ investigates the critical conditions for the ignition of an ALAS and the effect of the turbulence on the formation of the ignition sites. The manuscript [5] provides the experimental data on the effect of swirl flow on the length of the reverse flow zone, temperature and length of the flame. The region of the aluminum powder stable combustion in a high-speed airflow is determined in [6]. The authors of the study have obtained the dependence of the flame blow off velocity in the aluminum-air mixture flow on the value of the excess air ratio.

Swirling combustion is currently one of the most important engineering problems for the combustion physics. There is a hypothesis about an increase in the combustion efficiency of reacting gas mixtures in combustion chambers with swirling flow [7], as well as an increase in the efficiency of the fuel combustion devices due to swirling [8 - 11]. It is believed that swirling leads to the intensification of the heat and mass transfer and, accordingly, to an increase in the efficiency of the gas-dispersed fuel combustion. Usually to simulate the swirling flows, the Reynolds equations [9] or the Navier-Stokes equations [7] are used.

Our previous studies $[12,13]$ are dedicated to the combustion problems of the mixed solid fuels under a change in pressure [12] and under the high-speed blowing [13]. The present study is devoted to the combustion problem of the high-energy materials in the high-speed swirl flows. The interest of such problem is associated with the development of new methods to improve the efficiency of the powerproducing units. In this paper we have investigated the features of the ALAS flow and its combustion in a cylindrical channel with a sudden expansion and under the swirling conditions at the channel inlet. This problem is of practical importance, since, depending on the parameters of the suspension and swirl flow, stagnant zones can form in the cylindrical channel during the combustion (zones of particle accumulation 
or particle-free zones). In case of power-producing units, the irregularity of the particle distribution along the flow can significantly affect the speed of flame propagation through the channel.

A sudden expansion of the cylindrical channel can lead to a change in the characteristics of the air suspension flow. Gas swirling at the inlet of the channel also significantly changes the nature of the air suspension flow. In contrast to the study [3], the formulation of the problem in the present paper is based on the approaches of the mechanics of the two-phase reaction media [14].

\section{Physics and mathematics model and solution method}

The problem of the aluminum particles-air suspension flow and its combustion in a cylindrical channel with a sudden expansion is solved. The problem formulation is based on the approaches of the mechanics of the two-phase reaction media [14]. The initial gas tangential velocity is set to take into account the swirl of the flow. It is assumed that the aluminum-air suspension with the given mass fraction of the aluminum particles, particle radius $r_{p}$, velocity along the axial direction $u_{x b}$, zero velocity along the radial direction and velocity $u_{\phi}$ along the tangential direction is supplied from the inlet on the left side of the channel. The schematic diagram of the cylindrical channel is shown in Figure 1 (the notations are: $R$ is the radius of the channel at the outlet, $r$ is the radius of the channel at the inlet, 1 is the length of the narrow section of the channel, $L$ is the total length of the cylindrical channel). The arrows show the direction of the suspension feeding into the channel.

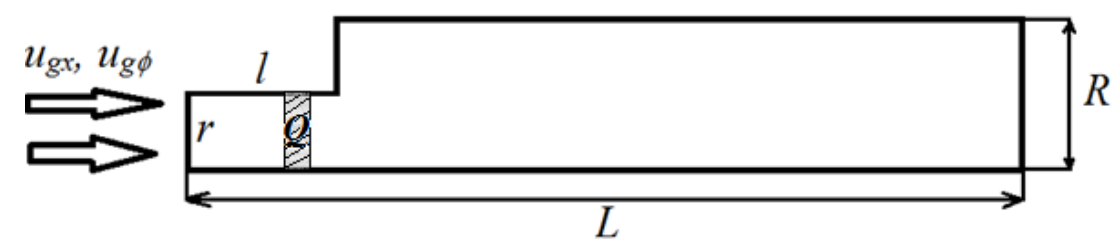

Fig.1. Scheme of the cylindrical channel with a sudden expansion/

Since the convective heat and mass transfer is much greater than the one by thermal conductivity and diffusion, the diffusion and heat conduction processes in gas phase are neglected. There is an ignition source with constant heat release inside the channel in the narrow section of the channel, schematically indicated by a shaded area (Figure 1). The rate of the heat release inside the chamber is equal to $Q$. The suspension flows through the chamber, heats up, and the heated aluminum particles start to ignite. The combustion mechanism of the aluminum particles is described in detail in [15 - 17]. The aluminum particles are supposed to ignite when they reach the ignition temperature $T_{p z}$. The rate of the heterogeneous chemical reaction on particle surface is defined taking into account the mass transfer. It is assumed that during the combustion of an aluminum particle, the radius of the melted aluminum in the particle $r_{A l}$ decreases, while as the radius of the whole particle $r_{p}$ grows due to the forming oxide layer. The thermal and dynamic interactions between aluminum particles and gas phase are taken into account under the assumption of the model. The developed mathematical model under the made assumption has the following form:

gas mass-conservation equation:

$$
\frac{\partial r \rho_{g}}{\partial t}+\frac{\partial r \rho_{g} u_{g}}{\partial x}+\frac{\partial r \rho_{g} v_{g}}{\partial r}=-r G
$$

gas impulse-conservation equation:

$$
\begin{aligned}
& \frac{\partial r \rho_{g} u_{g}}{\partial t}+\frac{\partial r\left(p_{g}+\rho_{g} u_{g}^{2}\right)}{\partial x}+\frac{\partial r \rho_{g} u_{g} v_{g}}{\partial r}=-r \tau_{x}-r G u_{g}, \\
& \frac{\partial r \rho_{g} v_{g}}{\partial t}+\frac{\partial r \rho_{g} u_{g} v_{g}}{\partial x}+\frac{\partial r\left(p_{g}+\rho_{g} v_{g}^{2}\right)}{\partial r}=-r \tau_{r}-r G v_{g}+p_{g}+\rho w_{g}^{2}, \\
& \frac{\partial r \rho_{g} w_{g}}{\partial t}+\frac{\partial r \rho_{g} w_{g} u_{g}}{\partial x}+\frac{\partial r \rho_{g} w_{g} v_{g}}{\partial r}=-r \tau_{\phi}-r G w_{g}-\rho_{g} w_{g} v_{g},
\end{aligned}
$$


oxygen mass-conservation equation:

$$
\frac{\partial r \rho_{O 2}}{\partial t}+\frac{\partial r \rho_{O 2} u_{g}}{\partial x}+\frac{\partial r \rho_{O 2} v_{g}}{\partial r}=-r G
$$

gas energy-conservation equation:

$$
\begin{aligned}
& \frac{\partial r \varepsilon_{g}}{\partial t}+\frac{\partial r u_{g}\left(\varepsilon_{g}+p_{g}\right)}{\partial x}+\frac{\partial r v_{g}\left(\varepsilon_{g}+p_{g}\right)}{\partial r}=-r G c_{g p} T_{g}+ \\
& r\left[u_{p} \tau_{x}+v_{p} \tau_{r}+w_{p} \tau_{\phi}+n_{p} \alpha_{p} S_{p}\left(T_{g}-T_{k}\right)-0.5 G\left(u_{g}^{2}+v_{g}^{2}+w_{g}^{2}\right)\right],
\end{aligned}
$$

particle mass-conservation equation:

$$
\frac{\partial r \rho_{p}}{\partial t}+\frac{\partial r \rho_{p} u_{p}}{\partial x}+\frac{\partial r \rho_{p} v_{p}}{\partial r}=r G
$$

particle impulse-conservation equation:

$$
\begin{aligned}
& \frac{\partial r \rho_{p} u_{p}}{\partial t}+\frac{\partial r \rho_{p} u_{p}^{2}}{\partial x}+\frac{\partial r \rho_{p} u_{p} v_{p}}{\partial r}=r \tau_{x}+r G u_{g}, \\
& \frac{\partial r \rho_{p} v_{p}}{\partial t}+\frac{\partial r \rho_{p} u_{p} v_{p}}{\partial x}+\frac{\partial r \rho_{p} v_{p}^{2}}{\partial r}=r \tau_{r}+r G v_{g}+\rho w_{p}^{2}, \\
& \frac{\partial r \rho_{p} w_{p}}{\partial t}+\frac{\partial r \rho_{p} w_{p} u_{p}}{\partial x}+\frac{\partial r \rho_{p} w_{p} v_{p}}{\partial r}=r \tau_{\phi}+r G w_{g}-\rho_{p} w_{p} v_{p},
\end{aligned}
$$

particle energy-conservation equation:

$$
\begin{aligned}
& \frac{\partial r \varepsilon_{p}}{\partial t}+\frac{\partial r u_{p} \varepsilon_{p}}{\partial x}+\frac{\partial r v_{p} \varepsilon_{p}}{\partial r}=r Q_{c h} G-r n_{p} \alpha_{p} S_{p}\left(T_{g}-T_{p}\right)- \\
& -r\left[u_{p} \tau_{x}+v_{p} \tau_{r}+w_{p} \tau_{\phi}+G c_{g p} T_{g}+0.5 G\left(u_{p}^{2}+v_{p}^{2}+w_{p}^{2}\right)\right],
\end{aligned}
$$

particles number equation:

$$
\frac{\partial r n_{p}}{\partial t}+\frac{\partial r n_{p} u_{p}}{\partial x}+\frac{\partial r n_{p} v_{p}}{\partial r}=0
$$

the gas equation:

$$
p_{g}=\rho_{g} T_{g} R_{g},
$$

initial conditions:

$$
\begin{aligned}
& u_{g}(x, r, 0)=v_{g}(x, r, 0)=w_{g}(x, r, 0)=u_{p}(x, r, 0)=v_{p}(x, r, 0)=w_{p}(x, r, 0)=0, \\
& n_{p}(x, r, 0)=\rho_{p}(x, r, 0)=0, T_{p}(x, r, 0)=T_{g}(x, r, 0)=T_{b}, \\
& \rho_{g}(x, r, 0)=\rho_{g b}, \rho_{O 2}(x, r, 0)=a_{O 2 b} \rho_{g b},
\end{aligned}
$$

Boundary conditions on the symmetry axis, $r=0$ : 


$$
\begin{aligned}
& \frac{\partial u_{g}(x, 0, t)}{\partial r}=\frac{\partial u_{p}(x, 0, t)}{\partial r}=\frac{\partial v_{g}(x, 0, t)}{\partial r}=\frac{\partial v_{p}(x, 0, t)}{\partial r}=0, \\
& \frac{\partial w_{g}(x, 0, t)}{\partial r}=\frac{\partial w_{p}(x, 0, t)}{\partial r}=0, \frac{\partial n_{p}(x, 0, t)}{\partial r}=\frac{\partial \rho_{p}(x, 0, t)}{\partial r}=0, \\
& \frac{\partial T_{p}(x, 0, t)}{\partial r}=\frac{\partial T_{g}(x, 0, t)}{\partial r}=\frac{\partial \rho_{g}(x, 0, t)}{\partial r}=\frac{\partial \rho_{O 2}(x, 0, t)}{\partial r}=0 .
\end{aligned}
$$

The following initial conditions (16) are set at the inlet of the channel $(x=0)$ : the enthalpy, the gas and particle flow rate, the axial, radial and tangential velocity of the gas and particles, the mass concentration of the oxidizer in the gas and the number of particles.

$$
\begin{gathered}
T_{p}(0, r, t)=T_{g}(0, r, t)=T_{s t}, \rho_{g}(0, r, t)=\rho_{g b}, \rho_{p}(0, r, t)=\Delta_{p} \rho_{g b}, \\
a_{O 2}(0, r, t)=a_{O 2 b}, u_{g}(0, r, t)=u_{p}(0, r, t)=u_{x b}, v_{g}(0, r, t)=v_{p}(0, r, t)=0, \\
w_{g}(0, r, t)=w_{p}(0, r, t)=u_{\phi}=A_{z}(r / R) u_{x b}, n_{p}=\Delta_{p} \rho_{g b} /\left(V_{p} \rho_{p}^{0}\right) .
\end{gathered}
$$

Atmospheric pressure is assumed at the outlet of the channel $(x=L)$.

The notations in Eqs. (1) - (16): $\varepsilon_{g}=p_{g} /(\gamma-1)+0.5 \rho_{g}\left(u_{g}^{2}+v_{g}^{2}+w_{g}^{2}\right)$ is the gas total energy, $\varepsilon_{p}=c_{p} T_{p} \rho_{p}+0.5 \rho_{p}\left(u_{p}^{2}+v_{p}^{2}+w_{p}^{2}\right)$ is the particle total energy, $\alpha_{p}=N u_{p} \lambda_{g} /\left(2 r_{p}\right)-$ is the gas-particle heat exchange coefficient, $\gamma=c_{p g} / c_{v g}$ is the adiabatic exponent, $\tau_{x}, \tau_{r}, \tau_{\phi}$ are the friction force along axial, radial and tangential direction, respectively, $\rho$ is the density, $u, v, w-$ are the velocity components along axial, radial and tangential direction, respectively, $t$ is the time, $x, r$ are the coordinates, $p$ is the pressure, $T$ is the temperature, $A_{z}$ is the constant of the swirling law, $\Delta_{p}$ is the particle mass fraction in the mixture, $Q_{c h}$ is the reaction heat effect; $a_{O 2}$ - oxidizer mass fraction in the gas phase. Indexes: $b$ is for the initial conditions of the parameters; $p$ is for the particle parameters, $g$ - gas parameters, $\mathrm{O}_{2}-$ oxygen, $s t-$ parameters at the inlet.

The current values of the aluminum radius in the particle $r_{A l}$ and the whole particle radius $r_{p}$ are determined as in the studies $[16,17]$ and are defined from the expressions:

$$
\begin{aligned}
& r_{A l}=\sqrt[3]{\left(\frac{\mu_{A l}+3 / 2 \mu_{O}}{\mu_{A l}} r_{p b}^{3}-\frac{\rho_{p}}{(4 / 3) \pi n_{p} \rho_{p}^{0}}\right) \frac{2 \mu_{A l}}{3 \mu_{O}}}, \\
& r_{p}=\sqrt[3]{\frac{3 \rho_{p}}{4 \pi \rho_{p}^{0} n_{p}}},
\end{aligned}
$$

where $\mu_{O}, \mu_{A l}$ are the molar masses of oxygen and aluminum, respectively, $\rho_{p}^{0}$ is the density of aluminum, $r_{p b}$ is the initial radius of the aluminum particle. Deriving the Eg. (17), we have assumed that the alumina $\left(\mathrm{Al}_{2} \mathrm{O}_{3}\right)$ remaining on the particle surface as a spherical layer. This assumption is introduced to determine the current value of the aluminum mass in a particle during its combustion. The aluminum particle burning rate is defined from the experimental data [15]. Taking into account the diffusional resistance of the reaction, the mass change rate of aluminum in the particle is written as:

$$
G=\alpha_{1} n_{p} \rho_{p}^{0} S_{A l} \frac{k\left(a_{O 2,}, r_{A l}\right) \beta_{m}}{\left(k\left(a_{O 2,}, r_{A l}\right)+\beta_{m}\right)}
$$

where $k\left(a_{O 2,} r_{A l}\right)=k_{0} a_{O 2}^{0.9} / \sqrt{r_{A l}}$ is the combustion law of a single aluminum particle under the excess of the oxidizer, $S_{A l}=4 \pi r_{A l}^{2}$ is the area of the unreacted aluminum surface, $k_{0}$ is the reaction rate constant, $\beta_{m}=\lambda_{g}(T) N u_{D} /\left(c_{g} \rho_{g} r_{p}\right)$ is the particle mass-transfer coefficient. 
The friction force along the axial direction is determined from the expression $\tau_{x}=n_{p} F_{x}[14,15]$, where $F_{x}=C_{x} S_{m} \rho_{g}\left(u_{g}-u_{p}\right)\left|u_{g}-u_{p}\right| / 2$ is the interaction force of a single particle with gas along the axial direction, $C_{x}=24\left(1+0.15 \operatorname{Re}_{x}^{0.682}\right) / \operatorname{Re}_{x}$ is the friction coefficient, $\operatorname{Re}_{x}=2 \rho_{g} r_{p}\left|u_{g}-u_{p}\right| / \eta$ is the Reynolds number, $S_{m}$ is the midsection area, $\eta$ is the dynamic viscosity of the gas. The components of the friction force along the radial and tangential directions are determined in a similar way.

The developed mathematical model has been solved numerically using the S.K. Godunov's scheme and is based on algorithms $[18,19]$. The size of the computational grid cells along the axial and radial directions is equal to $10^{-3} \mathrm{~m}$. The time step is determined from the Courant stability criteria [19]

$$
\frac{1}{\Delta t}<\frac{1}{\Delta t_{x}}+\frac{1}{\Delta t_{r}}
$$

where $\Delta t_{x}=\frac{h_{x}}{\max \left[\left|u_{g}\right|+c_{g}\right]}, \Delta t_{x}=\frac{h_{r}}{\max \left[\left|v_{g}\right|+c_{g}\right]}, c_{g}$ is the sound velocity in gas.

The verification of the numerical solution for the equations system (1) - (16) and the developed computer program for the reliability has been carried out by calculating the adiabatic combustion temperature and controlling for the conservation laws of the mass and total energy of the gas. According to the verification, the calculation error is about $0.03 \%$.

\section{Results and discussion}

We have carried out the parametric investigation on the effect of the swirling on the establishment of the ALAS combustion front in the channel and on the completeness of the particle combustion. The variable parameters in the calculations are the size and mass concentration of the particles, the value of the axial and angular velocities of the aluminum-air suspension supplied to the channel. All the calculations have been conducted under the following thermophysical and kinetic parameters borrowed from [17]: $Q=36.6 \mathrm{MJ} / \mathrm{kg}, k_{0}=1.11 \cdot 10^{-6} \mathrm{~m}^{1.5} / \mathrm{s}, \quad \alpha_{1}=0.889, \quad \lambda_{s t}=0.025 \mathrm{~W} /(\mathrm{m} \cdot \mathrm{K}), \quad T_{b}=300 \mathrm{~K}$, $\mu_{A l}=27 \cdot 10^{-3} \mathrm{~kg} / \mathrm{mol}, \quad \mu_{O}=16 \cdot 10^{-3} \mathrm{~kg} / \mathrm{mol}, \quad c_{p g}=1065 \mathrm{~J} /(\mathrm{kg} \cdot \mathrm{K}), \quad c_{v g}=768.2 \mathrm{~J} /(\mathrm{kg} \cdot \mathrm{K})$, $c_{p}=904 \mathrm{~J} /(\mathrm{kg} \cdot \mathrm{K}), \quad \rho_{p}^{0}=2380 \mathrm{~kg} / \mathrm{m}^{3}, \quad \eta=2 \cdot 10^{-5} \mathrm{~Pa} \cdot \mathrm{s}, \quad \rho_{O 2, b}=0.264 \mathrm{~kg} / \mathrm{m}^{3}, \quad p_{b}=0.1 \mathrm{MPa}$, $\rho_{b}=p_{b} / R_{g} T_{b}, T_{p z}=1400 \mathrm{~K}, r=0.02 \mathrm{~m}, R=0.1 \mathrm{~m}, \mathrm{~L}=0.3 \mathrm{~m}, \mathrm{l}=0.03 \mathrm{~m}$.

The results of some calculations are presented in Figures 2-9. Figures 2 - 9 are plotted for the steadystate regimes of the ALAS flow and its combustion. Figure 2 shows the stationary combustion regime for the ALAS in the case when the swirling is implemented according to the law $w_{g}(0, r, t)=A_{z}(r / R) u_{x b}$, where $R$ is the radius of the cylindrical channel, $A_{z}$ is the coefficient in the swirling law, $u_{x b}$ is the axial feed velocity. The calculation has been performed for the suspension with the axial feed velocity $u_{x b}=1 \mathrm{~m} / \mathrm{s}$, under the coefficient of the angular component of the flow velocity, $A_{z}=75$, under the mass concentration of the particles $-\rho_{p b}=0.1 \mathrm{~kg} / \mathrm{m}^{3}$, and with particle radius $r_{p b}=5 \cdot 10^{-6} \mathrm{~m}$. Figure 2a shows the gas temperature in the channel, Figure $2 \mathrm{~b}$ shows the mass concentration of the suspension. Under the selected swirl coefficient, the maximum angular velocity of the air suspension is $u_{\varphi, \max }=1.5 \mathrm{~m} / \mathrm{s}$.
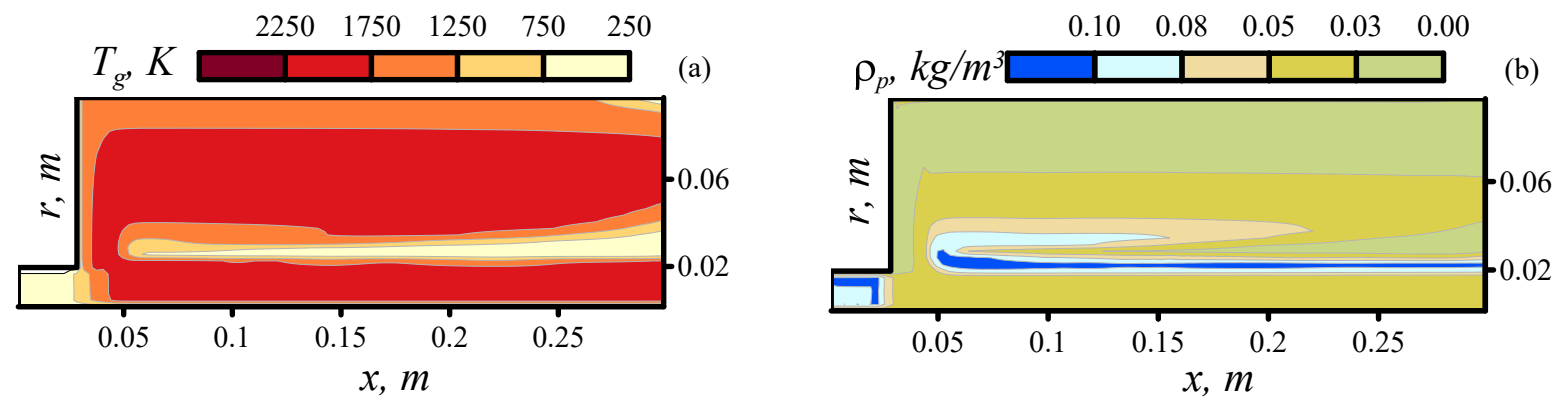

Fig.2. Gas temperature (a), mass concentration of the suspension (b), $\rho_{p b}=0.1 \mathrm{~kg} / \mathrm{m}^{3}, r_{p}=5 \cdot 10^{-6} \mathrm{~m}, u_{b}=1 \mathrm{~m} / \mathrm{s}, A_{z}=75$. 
In this case, the maximum concentration of the particles is observed at close range from the inlet of the channel at the height corresponding to the width of the inlet. The region of the maximum gas temperature is straight after the channel expand at $x=0.04 \mathrm{~m}$. With an increase in the particle mass concentration of the supplied mixture (Figure 3), the maximum temperature reached in the channel rises. The shape of the flame front remains practically unchanged. From a comparison of Figures $2 \mathrm{a}$ and $3 \mathrm{a}$, it can be seen that the heated gas zone stretches along the channel radius and slightly curves. Under the selected ratios of the axial and angular components of the mixture feed velocity at the inlet, the particles enter the combustion zone, ignite immediately at the entrance to the expanding part of the channel, and then are carried downstream by the flow. The main mass of the particles is concentrated along the mixture feed line (Figures 2b, 3b).

Figures 4, 5 show the calculation results in the case of a decrease in the mass concentration of aluminum particles in the supplied mixture. The coefficient of the angular component of the feed velocity in this calculation is $A_{z}=50$, thus the maximum value of the angle velocity is $1 \mathrm{~m} / \mathrm{s}$. Figure 4a shows the gas temperature under the mass concentration of the aluminum particles $\rho_{p b}=0.05 \mathrm{~kg} / \mathrm{m}^{3}$, Figure $5 \mathrm{a}$ $-\rho_{p b}=0.075 \mathrm{~kg} / \mathrm{m}^{3}$. Figures $4 \mathrm{~b}, 5 \mathrm{~b}$ show the distributions of the mass concentration of the particles under $\rho_{p b}=0.05 \mathrm{~kg} / \mathrm{m}^{3}$ and $\rho_{p b}=0.075 \mathrm{~kg} / \mathrm{m}^{3}$. Under the chosen calculation parameters, the shape of the combustion front and the spatial distribution of the particles change.
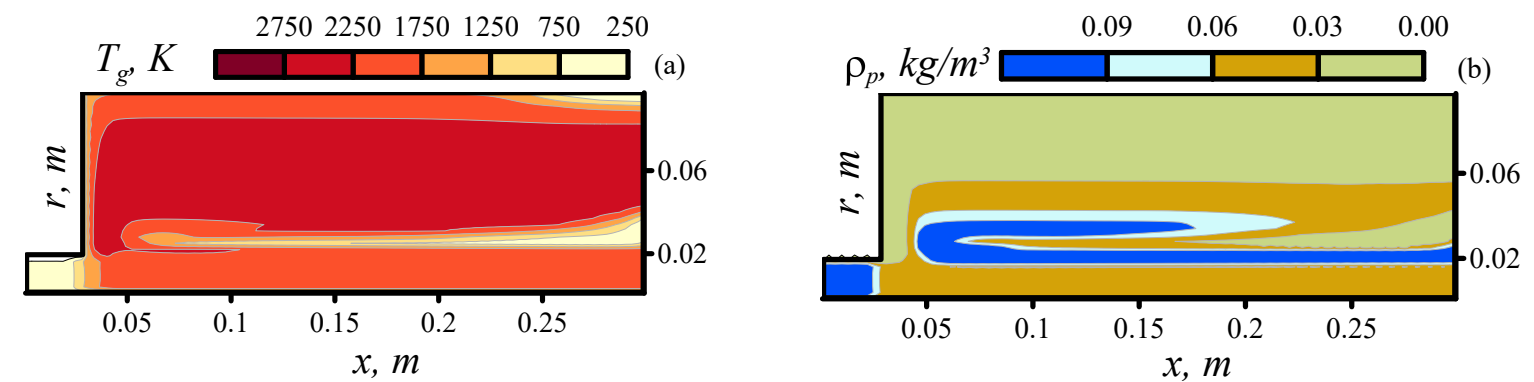

Fig.3. Gas temperature (a), mass concentration of the suspension (b), $\rho_{p b}=0.15 \mathrm{~kg} / \mathrm{m}^{3}, r_{p}=5 \cdot 10^{-6} \mathrm{~m}, u_{b}=1 \mathrm{~m} / \mathrm{s}, A_{z}=75$.
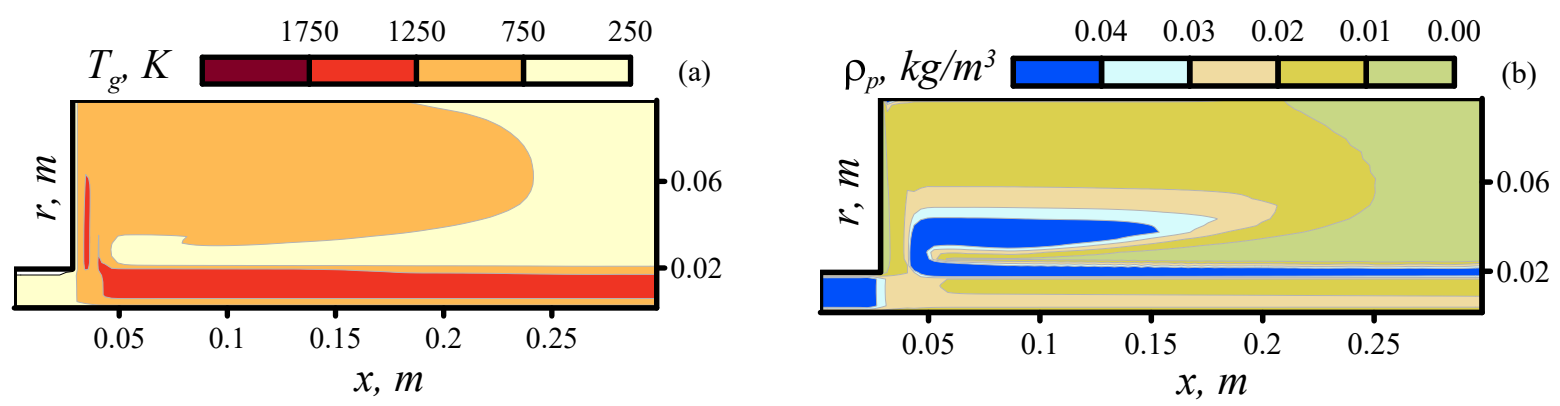

Fig.4. Gas temperature (a), mass concentration of the suspension (b), $\rho_{p b}=0.05 \mathrm{~kg} / \mathrm{m}^{3}, r_{p}=5 \cdot 10^{-6} \mathrm{~m}, u_{b}=1 \mathrm{~m} / \mathrm{s}, A_{z}=50$.
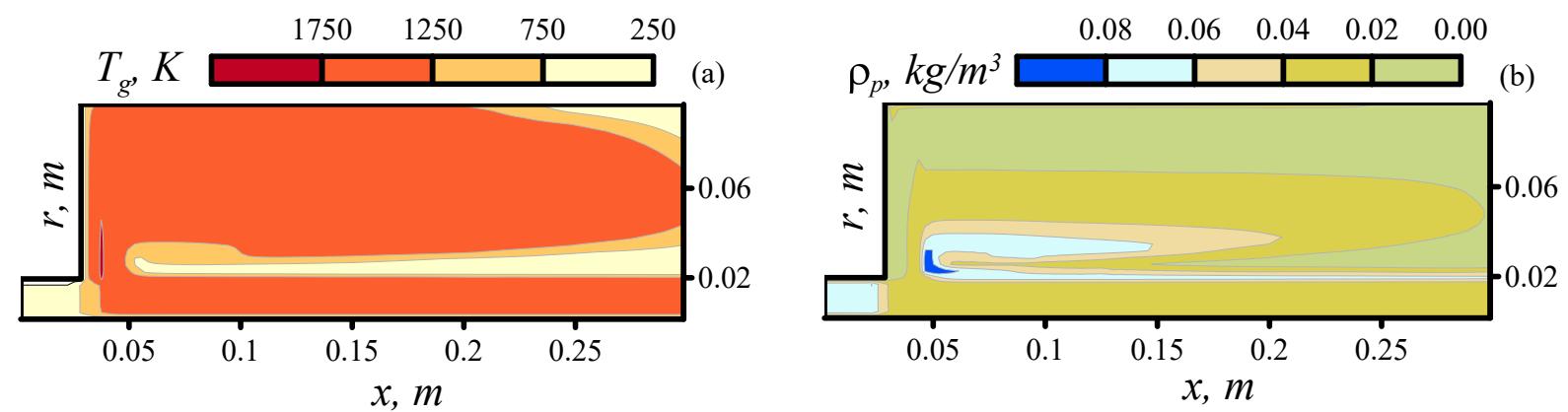

Fig. 5. Gas temperature (a), mass concentration of the suspension (b), $\rho_{p b}=0.075 \mathrm{~kg} / \mathrm{m}^{3}, r_{p}=5 \cdot 10^{-6} \mathrm{~m}, u_{b}=1 \mathrm{~m} / \mathrm{s}, A_{z}=50$. 
In the case of the low mass concentrations of the aluminum powder and high angular velocities of the supplied mixture at the inlet, the formation of the combustion front is observed along the axial direction of the mixture supply. The edge of the front corresponds to the width of the input channel (Figure 6). Figure 7 shows the particle velocity vector corresponding to the case plotted in Figure 6. Due to the high feed rate and high angular velocity component, the flame front is formed at a distance from the inlet channel at $x=0.06 \mathrm{~m}$. Qualitatively similar results had been obtained earlier in [20], where we had investigated the combustion of the coal dust-air suspension in a channel under the swirling flow conditions. In the study, we had conducted the numerical investigation on the influence of the flow swirling velocity on the gas temperature distribution inside the channel without expansion and on the completeness of the coal dust combustion. It had been shown that a particle with the low inertia is carried away by the swirling flow.
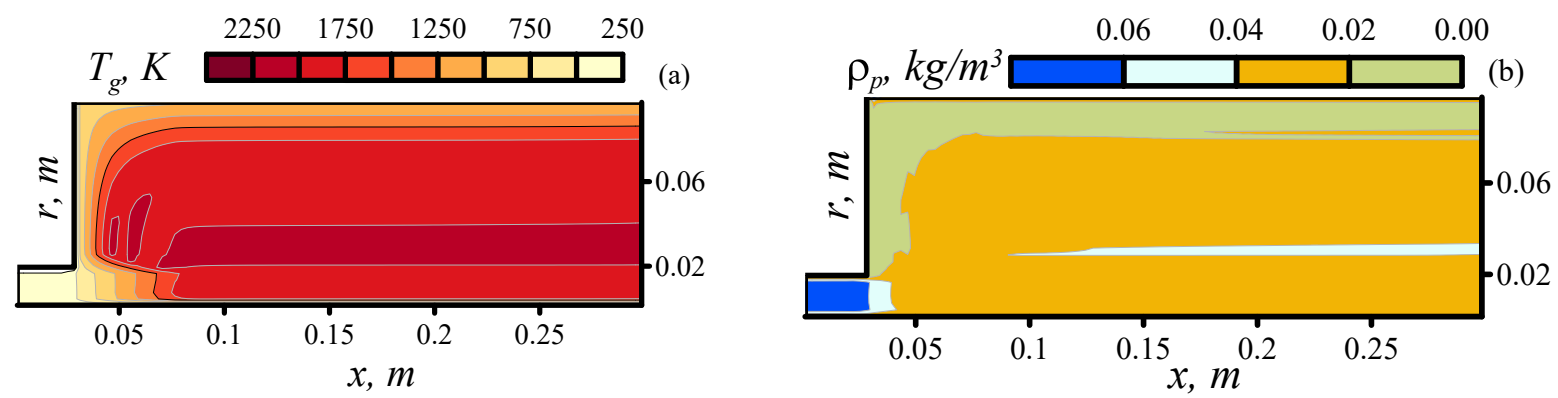

Fig.6. Gas temperature (a), mass concentration of the suspension (b), $\rho_{p b}=0.075 \mathrm{~kg} / \mathrm{m}^{3}, r_{p}=5 \cdot 10^{-6} \mathrm{~m}, u=3 \mathrm{~m} / \mathrm{s}, A_{z}=50$

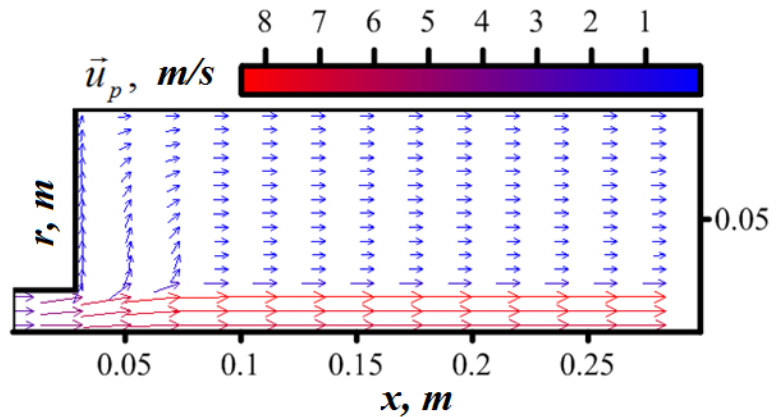

Fig.7. Velocity vector of the particle motion, $\rho_{p b}=0.075 \mathrm{~kg} / \mathrm{m}^{3}, r_{p}=5 \cdot 10^{-6} \mathrm{~m}, u_{b}=3 \mathrm{~m} / \mathrm{s}, A_{z}=50$.
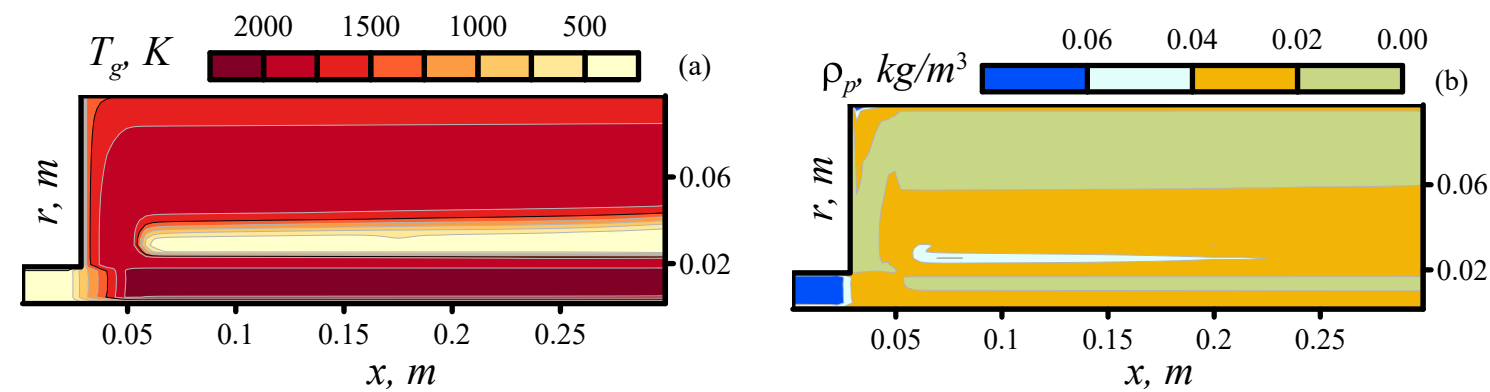

Fig.8. Gas temperature (a) and mass concentration of the suspension (b), $\rho_{p b}=0.075 \mathrm{~kg} / \mathrm{m}^{3}, r_{p}=10^{-6} \mathrm{~m}, u_{b}=10 \mathrm{~m} / \mathrm{s}, A_{z}=75$.

To study the effect of the aluminum particle size on the shape of the flame in the channel, we have conducted several calculation of the ALAS combustion with different particle radii ranged from 10 to 1 $\mu \mathrm{m}$. The results of two example calculation are shown in Figures 8, 9. Figure 8 shows the gas temperature and mass concentration of the aluminum powder along the channel during the ALAS combustion under the mass concentration $\rho_{p b}=0.075 \mathrm{~kg} / \mathrm{m}^{3}$, particle radius $10^{-6} \mathrm{~m}$, the feed velocity $u_{b}=10 \mathrm{~m} / \mathrm{s}$, and the coefficient of the angular component $A_{z}=75$. The intense swirl of the feed flow leads to a lifting of a 
certain fraction of the particles closer to the wall of the wide part of the channel. According to the Figure 8 , the combustion front is formed along the central part of the channel and in the area near the wall of the wide part of the channel.
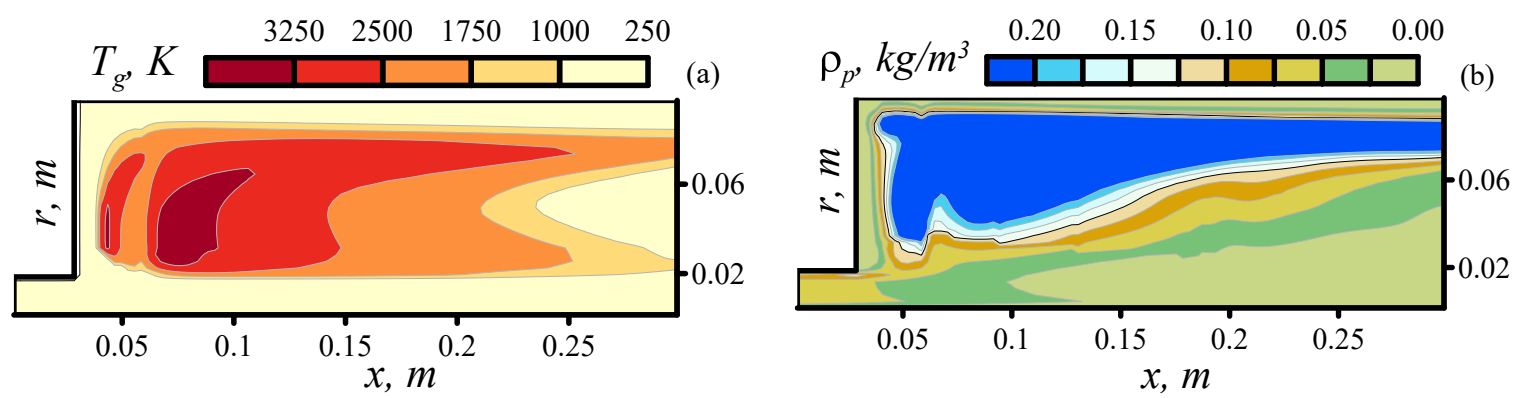

Fig.9. Gas temperature (a) and mass concentration of the suspension (b), $\rho_{p b}=0.075 \mathrm{~kg} / \mathrm{m}^{3}, r_{p}=10^{-5} \mathrm{~m}, u_{b}=5 \mathrm{~m} / \mathrm{s}, A_{z}=150$.

Figure 9 shows the calculation results of the ALAS combustion with the particle radius of $10 \mu \mathrm{m}$, under the particle mass concentration of $0.075 \mathrm{~kg} / \mathrm{m}^{3}$, the feed velocity $u_{b}=5 \mathrm{~m} / \mathrm{s}$, and the coefficient of the angular component $A_{z}=150$. The maximum speed along the tangential direction for Figures 8 and 9 was $150 \mathrm{~m} / \mathrm{s}$. The particles with the radius of $10 \mu \mathrm{m}$ have a sufficiently high inertia, which leads to their lag behind the gas and the formation of the zones of the increased particle concentration close to the channel walls. This effect provokes the formation of a curved flame front with the temperature maximum distant from the channel walls.

\section{Conclusion}

Thus, a physical and mathematical model has been developed for the combustion of an air suspension of aluminum powder in a channel with a sudden expansion during swirling of the flow. The model is based on the approaches of the mechanics of two-phase reacting media of R. I. Nigmatulin, and is written in a two-dimensional axisymmetric approximation, taking into account the swirl of the flow along the angular coordinate. The system of equations of the mathematical model is solved numerically by S.K. Godunov and A.N. Kraiko. Verification of the numerical solution of the system of equations showed that the laws of conservation of mass and total energy are satisfied with an accuracy of $99.97 \%$.

The influence of the value of the angular velocity of swirling of the flow at the entrance to the combustion chamber, the mass concentration and size of particles in the air suspension on the shape of the combustion front and the completeness of combustion of aluminum particles is studied numerically. The critical values of the swirling speed, leading to the sowing of aluminum particles onto the walls of the combustion chamber, have been determined. It is shown that, depending on the swirl rate of the air suspension feed at the entrance to the combustion chamber, the combustion front can be formed in the vicinity of the feed zone or be elongated along the channel axis. At high swirling speeds, part of the burning particles is thrown behind the channel turn, which leads to the curvature of the flame front, a two-front combustion mode arises, when the combustion fronts are formed in the center and at the periphery, between the center and the stacks of the burner. The developed physical and mathematical model of combustion of an air suspension of aluminum powder in a channel with a sudden expansion can be used for a preliminary analysis of the design parameters of burners on powder metal fuel.

\section{Acknowledgements \\ This study was carried out under the financial support of the Ministry of Education and Science of the Russian Federation, No 0721-2020-0036.}

\section{REFERENCES}

1 Egorov A.G. Combustion of dispersed aluminum in a stream of air: Monograph, SSC RAS, Samara, 2008, 305p. [in Russian]

2 Arkhipov V.A., Egorov A.G., Ivanin S.V., et al. Numerical simulation of aerodynamics and combustion of a gas mixture in a channel with sudden expansion. Combustion, Explosion and Shock Waves. 2010, Vol. 46, No. 6, pp. $647-655$. 
3 Egorov A.G. Thermal explosion of aluminum particles suspended in airflow. IOP Conference Series: Materials Science and Engineering. 2010, Vol. 734, No. 012189.

4 Egorov A.G., Pavlov D.A. Effect of initial turbulence on ignition, combustion, and flame stabilization in a gas-suspension flow. Combustion, Explosion and Shock Waves. 2006, Vol. 42, No. 1, pp. 32-40.

5 Egorov A.G., Tizilov A.S., Niyazov V.Y., et al. Effect of the swirl of cocurrent high-velocity air flow on the geometry of an aluminum-air flame. Russian Journal of the Physical Chemistry B. 2014, Vol. 8, No. 5, pp. 712715.

6 Tizilov A S., Egorov A.G. Limits of flame propagation in an aluminum-air mixture flow. Russian Journal of Physical Chemistry B. 2013, Vol. 7, No. 2, pp. 133-136.

7 Piralishvili S.A., Markovich D.M., Lobasov A.S., Vereshchagin I. M. Simulation of the working process of a supersonic combustor with a vortex igniter-stabilizer. Journal of Engineering Physics and Thermophysics. 2016, Vol. 89, No. 5, pp. 1325-1332.

8 Mikhailov A.S., Piralishvili S.A., Stepanov E.G., et al. Features of burning of pulverized peat fuel in a vortex burner device. Journal of Engineering Physics and Thermophysics. 2018, Vol. 91, No. 4, pp. 925-932.

9 Matvienko O.V., Bubenchikov A.N. Mathematical modeling of the heat transfer and chemical reaction of a swirling flow of a dissociative gas. Journal of Engineering Physics and Thermophysics. 2016, Vol. 89, No. 1, pp. 127-134.

10 Kuznetsov V.A., Dekterev A.A., Sentyabov A.V. Numerical study of devolatilization models on the combustion process of pulverized fuel flow swirling. Journal of Siberian Federal University. Engineering and Technologies. 2016, Vol. 9, No. 1, pp. 15-23.

11 Gavrilov A.A., Dekterev A.A., Sentyabov A.V. Modeling of swirling flows with coherent structures using the unsteady Reynolds stress transport model. Fluid Dynamics. 2015, Vol. 50, No. 4, pp. 471-482.

12 Krainov A., Poryazov V., Krainov D. Numerical simulation of the unsteady combustion of solid rocket propellants at a harmonic pressure change. Journal of Mechanical Science and Technology. 2020, Vol. 34, No. 1, pp. 489-497.

13 Krainov A., Poryazov V., Moiseeva K., Krainov D. Metalized solid propellant combustion under highspeed blowing flow. Journal of Mechanical Science and Technology. 2020, Vol. 34, No. 5, pp. 2245-2253.

14 Nigmatulin R.I. Dynamics of Multiphase Media. Moscow, 1987. [in Russian]

15 Belyaev A.F., Frolov Y.V., Korotkov A.I. Combustion and ignition of particles of finely dispersed aluminum. Combustion, Explosion, and Shock Waves. 1968, Vol. 4, No. 3, pp. $182-185$.

16 Poryazov V.A., Krainov A.Y., Krainov D.A. Simulating the combustion of $\mathrm{n}$ powder with added finely divided aluminum. Journal of Engineering Physics and Thermophysics. 2015, Vol. 88, No. 1, pp. 94-103.

17 Moiseeva K.M., Krainov A.Y., Dement'ev A.A. Critical conditions of spark ignition of a bidisperse aluminum powder in air. Combustion, Explosion, and Shock Waves. 2019, Vol. 55, No. 4, pp. 395-401.

18 Godunov S.K., Zabrodin A.V., Ivanov M.I., et al. Numerical solution of multidimensional problems of gas dynamics. Moscow, 1976. [in Russian]

19 Kraiko A.N. On discontinuity surfaces in a medium devoid of "proper" pressure. Journal of Applied Mathematics and Mechanics. 1979, Vol. 43, No. 3, pp. 539 - 549.

20 Moiseeva K.M., Krainov A.Y., Tarasenko Y.A. Combustion of a coal dust suspension in a pipe during the swirl. Journal of Physics: Conference Series. 2020, Vol. 1404, No. 1, pp. 012063. 